\title{
ON THE CLASSIFICATION AND REGULARITY OF UMBILIC SURFACES IN HOMOGENEOUS 3-MANIFOLDS
}

\author{
Rabah Souam $\quad$ Eric Toubiana
}

\begin{abstract}
We survey existence and classification of totally umbilic surfaces in the model geometries of Thurston and the Berger spheres. We also discuss the regularity of totally umbilic surfaces.
\end{abstract}

\section{Introduction}

The study of the geometry of surfaces in homogeneous 3-manifolds is a very active field nowadays, see for example [17], [5], [6], [20], [18], [14], [15], [13], [9], [3], [1], [12], [19], [4] and [8].

In the space forms the classification of totally umbilic surfaces is well known and very useful, see [23]. In $\mathbb{R}^{3}$ they are planes and round spheres and in $\mathbb{S}^{3}$ they are round spheres. In $\mathbb{H}^{3}$ they are totally geodesic planes and their equidistants, horospheres and round spheres.

In this paper we will survey the classification of totally umbilic surfaces in simply connected and homogeneous 3-manifolds obtained in [22].

Previously, the only known result in this direction is the non-existence of totally umbilic surfaces in the Heisenberg space due to A. Sanini, see [20].

We will first consider the Sol group, which has a 3-dimensional isometry group. Afterwards we will consider the manifolds having a 4-dimensional isometry group, denoted by $\mathbb{M}^{3}(\kappa, \tau)$ (see section 2 ). Namely these manifolds are

1991 Mathematics Subject Classification. 53C30, 53B25

Key words and phrases. totally umbilic, totally geodesic, homogeneous 3-manifolds. 
$\mathbb{S}^{2}(\kappa) \times \mathbb{R}(\kappa>0, \tau=0), \mathbb{H}^{2}(\kappa) \times \mathbb{R}(\kappa<0, \tau=0)$, the Berger spheres $(\kappa>0, \tau \neq 0)$ and the manifolds having the isometry group of either the Heisenberg space $(\kappa=0, \tau \neq 0)$ or $\widetilde{\mathrm{PSL}_{2}(\mathbb{R})}(\kappa<0, \tau \neq 0)$, see [2], [21] or [24]. Except for the Berger spheres, these manifolds are five of the eight model geometries of Thurston [24]. The remaining model geometries are the three space forms.

Throughout this paper all the surfaces are assumed connected and of class $C^{3}$. In this regard we observe that the standard proof of the classification of umbilic hypersurfaces in the space forms assumes they are of class $C^{3}$. We will end this paper showing that in fact it is enough to assume the hypersurfaces are twice differentiable. Consequently this is also the case in $\mathbb{H}^{2} \times \mathbb{R}$ and $\mathbb{S}^{2} \times \mathbb{R}$, see remark 11.

We are grateful to the referee for valuable observations.

\section{Classification of totally umbilic surfaces in homogeneous 3-manifolds}

It is known that the isometry group of any simply connected and homogeneous Riemannian 3-manifold is a Lie group of dimension 3, 4 or 6, see [2], [21] and [24].

If the dimension is 6 then the manifold is a space form.

Let us consider the case where the dimension is 4 . Such a manifold is a fibration over some simply connected and complete surface, $\mathbb{M}^{2}(\kappa)$, of constant curvature $\kappa \in \mathbb{R}$, with geodesic fibers. Actually, for each $\kappa$, there is a one-parameter family $\mathbb{M}^{3}(\kappa, \tau)$ of such fibrations, parametrized by the bundle curvature $\tau \in \mathbb{R}$, see [24]. When $\tau=0$ then $\mathbb{M}^{3}(\kappa, 0)$ is just a Riemannian product $\mathbb{M}^{2}(\kappa) \times \mathbb{R}$.

The unit vector field $\xi$ tangent to the fibers is a Killing field. The field $\xi$ defines the vertical direction of the Riemannian submersion $\mathbb{M}^{3}(\kappa, \tau) \rightarrow \mathbb{M}^{2}(\kappa)$.

Moreover we assume $\kappa-4 \tau^{2} \neq 0$, otherwise the manifold is a space form and its isometry group has dimension 6 . 
The manifolds $\mathbb{M}^{3}(\kappa, \tau)$ with $\tau \neq 0$ are of three types : when $\kappa>0$ they are the Berger spheres, for $\kappa=0$ they have the isometry group of the Heisenberg space, $\mathrm{Nil}_{3}$, and for $\kappa<0$ they have the isometry group of $\widetilde{\mathrm{PSL}_{2}(\mathbb{R})}$.

Let us assume now that the isometry group has dimension 3. If we suppose moreover that the manifold has a compact Riemannian quotient then, up to isometry, the manifold is unique and is a Lie group called the Sol group (or Sol geometry). A model of $S o l$ is $\mathbb{R}^{3}$ equipped with the metric:

$$
d s^{2}=e^{2 z} \mathrm{~d} x^{2}+e^{-2 z} \mathrm{~d} y^{2}+\mathrm{d} z^{2} .
$$

The group structure of $S o l$ is given by

$$
\left(x^{\prime}, y^{\prime}, z^{\prime}\right) \star(x, y, z)=\left(e^{-z^{\prime}} x+x^{\prime}, e^{z^{\prime}} y+y^{\prime}, z+z^{\prime}\right) .
$$

The isometries are:

$(x, y, z) \mapsto\left( \pm e^{-c} x+a, \pm e^{c} y+b, z+c\right)$ and $(x, y, z) \mapsto\left( \pm e^{-c} y+a, \pm e^{c} x+b,-z+c\right)$,

where $a, b$ and $c$ are any real numbers.

For more details we refer to [2], [21] and [24].

Let $X: \Omega \rightarrow \mathbb{M}$ be an immersion where $\Omega \subset \mathbb{R}^{2}$ is a connected open set and $\mathbb{M}$ stands for either $\mathbb{M}^{3}(\kappa, \tau)$ or Sol. We denote by $N$ a unit normal field along $X$. We assume the immersion $X$ is umbilic, this means there exists a real function $\lambda$ on $\Omega$ satisfying:

$$
\left\{\begin{array}{l}
\bar{\nabla}_{X_{u}} N=\lambda X_{u} \\
\bar{\nabla}_{X_{v}} N=\lambda X_{v}
\end{array}\right.
$$

where $\nabla$ is the connection on $\mathbb{M}$ and $u$ and $v$ are the coordinates on $\Omega$.

We set $\nu=\langle N, \xi\rangle$ and $T:=\xi-\nu N$ (respectively $\nu=\left\langle N, \frac{\partial}{\partial z}\right\rangle$ and $T:=$ $\left.\frac{\partial}{\partial z}-\nu N\right)$ in case where $\mathbb{M}=\mathbb{M}^{3}(\kappa, \tau)$ (respectively $\left.\mathbb{M}=S o l\right)$.

The starting idea in analyzing the totally umbilic surfaces is the following formula. 
Proposition 1 With the previous notations we have

$$
\nabla \lambda=\left\{\begin{aligned}
\left(\kappa-4 \tau^{2}\right) \nu T, & \text { if } \mathbb{M}=\mathbb{M}^{3}(\kappa, \tau) \\
2 \nu T, & \text { if } \mathbb{M}=S o l
\end{aligned}\right.
$$

where $\nabla \lambda$ stands for the gradient of $\lambda$ on the surface.

Proof. From relations (1) we derive the following

$$
\left\{\begin{array}{l}
\bar{\nabla}_{X_{v}}\left(\bar{\nabla}_{X_{u}} N\right)=\lambda_{v} X_{u}+\lambda \bar{\nabla}_{X_{v}} X_{u} \\
\bar{\nabla}_{X_{u}}\left(\bar{\nabla}_{X_{v}} N\right)=\lambda_{u} X_{v}+\lambda \bar{\nabla}_{X_{u}} X_{v}
\end{array}\right.
$$

Substracting the second equation from the first one we get

$$
\bar{\nabla}_{X_{v}}\left(\bar{\nabla}_{X_{u}} N\right)-\bar{\nabla}_{X_{u}}\left(\bar{\nabla}_{X_{v}} N\right)=\lambda_{v} X_{u}-\lambda_{u} X_{v}
$$

That is

$$
R\left(X_{u}, X_{v}\right) N=\lambda_{v} X_{u}-\lambda_{u} X_{v},
$$

where $R$ denotes the curvature tensor of $\mathbb{M}$.

In case where $\mathbb{M}=\mathbb{M}^{3}(\kappa, \tau)$ we have the following formula derived by Daniel, see $[6]$ :

$$
R\left(X_{u}, X_{v}\right) N=\left(\kappa-4 \tau^{2}\right) \nu\left(\left\langle X_{v}, T\right\rangle X_{u}-\left\langle X_{u}, T\right\rangle X_{v}\right)
$$

Taking into account the relation (2) we get

$$
\nabla \lambda=\left(\kappa-4 \tau^{2}\right) \nu T
$$

as desired.

Now if $\mathbb{M}=S o l$ we can prove as in [6] (see [22] for details):

$$
R\left(X_{u}, X_{v}\right) N=2 \nu\left(\left\langle X_{v}, T\right\rangle X_{u}-\left\langle X_{u}, T\right\rangle X_{v}\right)
$$

From what we deduce using (2):

$$
\nabla \lambda=2 \nu T
$$

which concludes the proof.

The classification of totally umbilic surfaces in $\mathbb{M}$ is summarized in what follows. 
Theorem 2 Up to ambient isometries, there exist only two complete totally umbilic surfaces in Sol, one of them being totally geodesic and the other not. Both surfaces are invariant under the horizontal translations $(x, y, z) \mapsto(x+$ $t, y, z), t \in \mathbb{R}$. The totally geodesic surface corresponds to the plane $\{y=0\}$. The non geodesic one is a vertical graph contained in a slab delimited by two totally geodesic planes $\left\{y= \pm y_{0}\right\}, y_{0}>0$, and is asymptotic to them.

Moreover, up to ambient isometries, any totally umbilic surfaces in Sol is contained in one of these two surfaces.

Theorem 3 There exist no totally umbilic surfaces (even non complete) in the three manifolds $\mathbb{M}^{3}(\kappa, \tau)$, with $\tau \neq 0$ and $\kappa-4 \tau^{2} \neq 0$. In particular, there are no totally geodesic surfaces.

Now we consider the case where $\mathbb{M}=\mathbb{M}^{3}(\kappa, 0)$ that is $\mathbb{M}=\mathbb{M}^{2}(\kappa) \times \mathbb{R}$, $\kappa \in \mathbb{R}$. When $\kappa=0$ we recover the Euclidean space $\mathbb{R}^{3}$ so we will assume $\kappa \neq 0$. Without loss of generality we can further consider only the cases $\kappa= \pm 1$.

It is easily seen that the isometries of $\mathbb{M}^{2}(\kappa) \times \mathbb{R}$ are of the form

$$
(p, z) \in \mathbb{M}^{2}(\kappa) \times \mathbb{R} \mapsto(\varphi(p), \pm z+a)
$$

where $\varphi$ is an isometry of $\mathbb{M}^{2}(\kappa)$ and $a \in \mathbb{R}$.

Let us consider the one-parameter groups of isometries $\left\{\varphi_{t}, t \in \mathbb{R}\right\}$ in $\mathbb{M}^{2}(\kappa)$ :

1. if $\kappa=1$, that is $M^{2}(1)=\mathbb{S}^{2}$, it is just the group of rotations around a fixed point of $\mathbb{S}^{2}$,

2. If $\kappa=-1$, that is $M^{2}(-1)=\mathbb{H}^{2}$, there are three cases:

(a) $\left\{\varphi_{t}, t \in \mathbb{R}\right\}$ is the group of rotations around a fixed point of $\mathbb{H}^{2}$,

(b) $\left\{\varphi_{t}, t \in \mathbb{R}\right\}$ is the group of hyperbolic translations along a geodesic of $\mathbb{H}^{2}$,

(c) $\left\{\varphi_{t}, t \in \mathbb{R}\right\}$ is the group of parabolic isometries of $\mathbb{H}^{2}$ with respect to a fixed point of the asymptotic boundary of $\mathbb{H}^{2}$. 
Definition 4 We say that a surface $S$ in $\mathbb{M}^{2}(\kappa) \times \mathbb{R}$ is symmetric if it is invariant under a one-parameter group of isometries of the form: $(p, z) \mapsto\left(\varphi_{t}(p), z\right)$ where $\left\{\varphi_{t}, t \in \mathbb{R}\right\}$ is as above.

We first state:

Theorem 5 Any umbilic surface of $\mathbb{M}^{2}(\kappa) \times \mathbb{R}$ is part of a symmetric umbilic surface.

We just sketch the proof. From proposition 1 we have $\nabla \lambda=\kappa \nu T$, which shows the level curves of $\lambda$ are horizontal. Since the horizontal slices $\mathbb{M}^{2}(\kappa) \times$ $\left\{z_{0}\right\}$ are totally geodesic, we deduce that the angle between the surface and each slice is locally constant. A simple computation then shows that each level curve has constant geodesic curvature in $\mathbb{M}^{2}(\kappa)$. Using these two properties it can be shown that the surface is part of a symmetric surface (see [22] for more details).

As a consequence of Theorem 5, classifying umbilic surfaces of $\mathbb{M}^{2}(\kappa) \times \mathbb{R}$ reduces to classifying the symmetric ones. This is summarized in what follows.

Theorem 6 Any totally geodesic surface in $\mathbb{M}^{2}(\kappa) \times \mathbb{R}$ is part of a complete one.

The only complete totally geodesic surfaces in $\mathbb{M}^{2}(\kappa) \times \mathbb{R}$ are the slices $\mathbb{M}^{2}(\kappa) \times\left\{z_{0}\right\}$ and the products $\Gamma \times \mathbb{R}$ where $\Gamma$ is a complete geodesic in $\mathbb{M}^{2}(\kappa)$.

Theorem 7 Any totally umbilic surface in $\mathbb{S}^{2} \times \mathbb{R}$ is part of a complete one.

Up to ambient isometries, there are three types of complete and totally umbilic surfaces in $\mathbb{S}^{2} \times \mathbb{R}$, besides the totally geodesic ones:

1. a one-parameter family of embedded topological spheres homologous to zero 
2. a one-parameter family of embedded topological spheres nonhomologous to zero

3. a unique properly embedded surface homeomorphic to $\mathbb{R}^{2}$.

Moreover all these surfaces are symmetric.

Theorem 8 Any totally umbilic surface in $\mathbb{H}^{2} \times \mathbb{R}$ is part of a complete one.

Up to ambient isometries, there are three types of complete and totally umbilic surfaces in $\mathbb{H}^{2} \times \mathbb{R}$, besides the totally geodesic ones:

1. a one-parameter family of embedded topological spheres invariant under a one-parameter group of isometries of the form $\left(\varphi_{t}, I d\right), t \in \mathbb{R}$, where $\left\{\varphi_{t}, t \in \mathbb{R}\right\}$ is the group of rotations around a fixed point of $\mathbb{H}^{2}$

2. a one-parameter family of properly embedded surfaces homeomorphic to $\mathbb{R}^{2}$ invariant under a one-parameter group of isometries of the form $\left(\varphi_{t}, I d\right)$, $t \in \mathbb{R}$, where $\left\{\varphi_{t}, t \in \mathbb{R}\right\}$ is the group of hyperbolic translations along a geodesic of $\mathbb{H}^{2}$

3. a unique properly embedded surface homeomorphic to $\mathbb{R}^{2}$ invariant under a one-parameter group of isometries of the form $\left(\varphi_{t}, I d\right), t \in \mathbb{R}$, where $\left\{\varphi_{t}, t \in \mathbb{R}\right\}$ is the group of parabolic isometries of $\mathbb{H}^{2}$ with respect to a fixed point of the asymptotic boundary of $\mathbb{H}^{2}$.

\section{Regularity of totally umbilic surfaces}

The standard proof of the classification of totally umbilic surfaces in $\mathbb{R}^{3}$ assumes that the surfaces have at least $C^{3}$ regularity, see for instance [7], [11], [16] or [23], Vol.3. However to define umbilic surfaces it is enough to assume twice differentiability, that is locally the surface is given by an immersion such that each coordinate function is differentiable as well as its first partial derivatives. 
We will show that in fact any totally umbilic surface in $\mathbb{R}^{3}$ which is twice differentiable is actually of class $C^{3}$ and so the classification is also valid with this weaker regularity. The fact that a totally umbilic $C^{2}$ surface in $\mathbb{R}^{3}$ is a piece of a round sphere or of a plane is probably known but we are not aware of any reference. For instance this problem was mentioned in the recent book of Kühnel ([16], p. 77). We note that the total umbilicity condition is a Weingarten relation but not of special type in the sense of Hartman and Wintner [10]. Therefore the techniques used in [10] for $C^{2}$ surfaces do not apply to totally umbilic surfaces.

Since changing (locally) conformally the metric of the ambient space preserves umbilicity (see [23], Vol.4), it follows that twice differentiability implies $C^{3}$ regularity in any locally conformally flat (smooth ) 3-manifold. In particular the classification of totally umbilic surfaces in $\mathbb{H}^{3}, \mathbb{S}^{3}, \mathbb{S}^{2} \times \mathbb{R}$ and $\mathbb{H}^{2} \times \mathbb{R}$ is valid assuming only twice differentiability, as these spaces are locally conformally flat, see Remark 11. We think this regularity result is true in any manifold.

Theorem 9 Let $S \subset \mathbb{R}^{3}$ be a twice differentiable totally umbilic surface. Then $S$ is in fact of class $C^{3}$. Consequently $S$ is part of a round sphere or a plane.

Proof. By assumption $S$ is locally the graph of a twice differentiable function $h$ defined on a open set $U \subset \mathbb{R}^{2}$. Therefore, $S$ is locally parametrized by:

$$
X(x, y)=(x, y, h(x, y)), \quad(x, y) \in U .
$$

A unit normal field is

$$
N=\frac{\left(-h_{x},-h_{y}, 1\right)}{\sqrt{1+h_{x}^{2}+h_{y}^{2}}}
$$

As $S$ is totally umbilic there exists a real function $\lambda$ on $U$ such that $N_{x}=\lambda X_{x}$ and $N_{y}=\lambda X_{y}$. Since

$$
\begin{aligned}
& N_{x}=\frac{1}{\left(1+h_{x}^{2}+h_{y}^{2}\right)^{3 / 2}}\left(-\left(1+h_{y}^{2}\right) h_{x x}+h_{x} h_{y} h_{y x}, h_{x} h_{y} h_{x x}-\left(1+h_{x}^{2}\right) h_{y x},-h_{x} h_{x x}-h_{y} h_{y x}\right) \\
& N_{y}=\frac{1}{\left(1+h_{x}^{2}+h_{y}^{2}\right)^{3 / 2}}\left(-\left(1+h_{y}^{2}\right) h_{x y}+h_{x} h_{y} h_{y y}, h_{x} h_{y} h_{x y}-\left(1+h_{x}^{2}\right) h_{y y},-h_{x} h_{x y}-h_{y} h_{y y}\right)
\end{aligned}
$$


we have

$$
N_{x}=\lambda X_{x} \Leftrightarrow\left(S_{1}\right)\left\{\begin{aligned}
-\left(1+h_{y}^{2}\right) h_{x x}+h_{x} h_{y} h_{y x} & =\lambda\left(1+h_{x}^{2}+h_{y}^{2}\right)^{3 / 2} \\
h_{x} h_{y} h_{x x}-\left(1+h_{x}^{2}\right) h_{y x} & =0 \\
-h_{x} h_{x x}-h_{y} h_{y x} & =\lambda h_{x}\left(1+h_{x}^{2}+h_{y}^{2}\right)^{3 / 2}
\end{aligned}\right.
$$

and

$$
N_{y}=\lambda X_{y} \Leftrightarrow\left(S_{2}\right)\left\{\begin{aligned}
-\left(1+h_{y}^{2}\right) h_{x y}+h_{x} h_{y} h_{y y} & =0 \\
h_{x} h_{y} h_{x y}-\left(1+h_{x}^{2}\right) h_{y y} & =\lambda\left(1+h_{x}^{2}+h_{y}^{2}\right)^{3 / 2} \\
-h_{x} h_{x y}-h_{y} h_{y y} & =\lambda h_{y}\left(1+h_{x}^{2}+h_{y}^{2}\right)^{3 / 2}
\end{aligned}\right.
$$

Up to a rotation and restricting the open set $U$ if necessary, we can assume that the first derivatives $h_{x}$ and $h_{y}$ never vanish on $U$.

From the last two equations of $\left(S_{1}\right)$ we obtain

$$
\begin{aligned}
& h_{x x}=-\lambda\left(1+h_{x}^{2}\right) \sqrt{1+h_{x}^{2}+h_{y}^{2}} \\
& h_{y x}=-\lambda h_{x} h_{y} \sqrt{1+h_{x}^{2}+h_{y}^{2}}
\end{aligned}
$$

In the same way, from $\left(S_{2}\right)$ we get

$$
\begin{aligned}
& h_{x y}=-\lambda h_{x} h_{y} \sqrt{1+h_{x}^{2}+h_{y}^{2}} \\
& h_{y y}=-\lambda\left(1+h_{y}^{2}\right) \sqrt{1+h_{x}^{2}+h_{y}^{2}}
\end{aligned}
$$

Thus

$$
\left\{\begin{aligned}
h_{x x} & =-\lambda\left(1+h_{x}^{2}\right) \sqrt{1+h_{x}^{2}+h_{y}^{2}} \\
h_{x y}= & h_{y x}=-\lambda h_{x} h_{y} \sqrt{1+h_{x}^{2}+h_{y}^{2}} \\
h_{y y} & =-\lambda\left(1+h_{y}^{2}\right) \sqrt{1+h_{x}^{2}+h_{y}^{2}}
\end{aligned}\right.
$$

Observe that the second equation in $\left(S_{1}\right)$ is equivalent to

$$
\frac{2 h_{x} h_{x x}}{1+h_{x}^{2}}=\frac{2 h_{y x}}{h_{y}} .
$$


This means that

$$
\frac{\partial}{\partial x}\left(\log \left(1+h_{x}^{2}\right)-\log h_{y}^{2}\right) \equiv 0 .
$$

Therefore

$$
\log \left(1+h_{x}^{2}\right)=\log h_{y}^{2}+f(y)
$$

where $f$ is a real function depending only on $y$. So

$$
1+h_{x}^{2}=e^{f(y)} h_{y}^{2} .
$$

In the same way, considering the first equation of $\left(S_{2}\right)$ we get

$$
1+h_{y}^{2}=e^{g(x)} h_{x}^{2},
$$

where $g$ is a real function depending only on $x$. We infer that

$$
\left(1+e^{f(y)}\right) \frac{h_{y}^{2}}{h_{x}^{2}}=1+e^{g(x)} .
$$

Equation (5) shows that $f$ is differentiable. Taking the derivatives in (6) with respect to $y$ and using the relations in (4) we obtain after some simplifications

$$
\lambda=\frac{f^{\prime}(y) e^{f(y)} h_{y}}{2\left(1+e^{f(y)}\right) \sqrt{1+h_{x}^{2}+h_{y}^{2}}}
$$

Analogously we get

$$
\lambda=\frac{g^{\prime}(x) e^{g(x)} h_{x}}{2\left(1+e^{g(x)}\right) \sqrt{1+h_{x}^{2}+h_{y}^{2}}}
$$

From the equations (7) and (8) we derive

$$
f^{\prime}(y)=\frac{\left(1+e^{f(y)}\right)}{e^{f(y)} h_{y}} \frac{g^{\prime}(x) e^{g(x)} h_{x}}{1+e^{g(x)}} .
$$

From (9) we see that $f^{\prime}$ is differentiable. Using (7) this shows the continuity of $\lambda$. The relations in (4) then imply the $C^{2}$ regularity of $h$. Coming back to (9) we find that $f$ is of class $C^{1}$. Consequently (7) gives that $\lambda$ is of class $C^{1}$. Finally we conclude using again the relations in (4) that $h$ has $C^{3}$ regularity. Consequently $S$ is of class $C^{3}$ and then the usual argument shows that $S$ is part of a round sphere or a plane. 
Remark 10 In fact the same proof works assuming only that $h$ is $C^{1}$ and has second partial derivatives. Moreover the relations involved in the proof of the theorem can be used to show that $h$ is in fact $C^{\infty}$.

Remark 11 It is clear that $\mathbb{S}^{3}$ and $\mathbb{H}^{3}$ are locally conformally flat since they admit local coordinates where their metric is proportional to the Euclidean metric. The spaces $\mathbb{H}^{2} \times \mathbb{R}$ and $\mathbb{S}^{2} \times \mathbb{R}$ are also locally conformally flat. More precisely we will show that $\left.\mathbb{H}^{2} \times\right] 0, \pi\left[\right.$ is conformally equivalent to $\mathbb{H}^{3}$ and $\mathbb{S}^{2} \times \mathbb{R}$ is conformally equivalent to $\mathbb{R}^{3}-\{(0,0,0)\}$.

Proof of Remark 11. First consider the space $\mathbb{H}^{2} \times \mathbb{R}$. Choose a geodesic plane $\mathbb{H}^{2}$ in $\mathbb{H}^{3}$ and choose a unit normal field $N$ along it. For each point $p \in \mathbb{H}^{2} \subset \mathbb{H}^{3}$ and for each $t \in \mathbb{R}$ consider the point at the signed distance $t$ from $p$ on the normal geodesic of $\mathbb{H}^{2}$ issued from $p$ and directed by $N(p)$. This defines a diffeomorphism $F$ from $\mathbb{H}^{2} \times \mathbb{R}$ onto $\mathbb{H}^{3}$. Then the pullback metric on $\mathbb{H}^{2} \times \mathbb{R}$ is $\mathrm{d} s^{2}=\cosh ^{2}(t) \mathrm{d} s_{\mathbb{H}^{2}}^{2}+\mathrm{d} t^{2}$. To see this we choose the halfspace models for $\mathbb{H}^{3}$ and $\mathbb{H}^{2}$, that is

$\mathbb{H}^{3}=\left\{(u, v, w) \in \mathbb{R}^{3}, w>0\right\}$, equipped with the metric $\mathrm{d} s_{\mathbb{H}^{3}}^{2}=\frac{\mathrm{d} u^{2}+\mathrm{d} v^{2}+\mathrm{d} w^{2}}{w^{2}}$

and

$$
\mathbb{H}^{2}=\left\{(x, y) \in \mathbb{R}^{2}, y>0\right\}, \text { equipped with the metric } \mathrm{d} s_{\mathbb{H}^{2}}^{2}=\frac{\mathrm{d} x^{2}+\mathrm{d} y^{2}}{y^{2}} .
$$

Then the diffeomorphism $F$ is given by $F(x, y, t)=\left(y \tanh t, x, \frac{y}{\cosh t}\right)$. A straightforward computation gives

$$
F^{*}\left(\frac{\mathrm{d} u^{2}+\mathrm{d} v^{2}+\mathrm{d} w^{2}}{w^{2}}\right)=\cosh ^{2} t\left(\frac{\mathrm{d} x^{2}+\mathrm{d} y^{2}}{y^{2}}\right)+\mathrm{d} t^{2} .
$$

With the new coordinate $z=2 \arctan \left(e^{t}\right)$ the metric reads as

$$
\mathrm{d} s^{2}=\frac{1}{\sin ^{2} z}\left(\mathrm{~d} s_{\mathbb{H}^{2}}^{2}+\mathrm{d} z^{2}\right)
$$

on $\left.\mathbb{H}^{2} \times\right] 0, \pi[$. 
In the case of $\mathbb{S}^{2} \times \mathbb{R}$ we consider the map

$$
\begin{aligned}
\left(\mathbb{S}^{2} \times \mathbb{R}, e^{2 t}\left(\mathrm{~d} s_{\mathbb{S}^{2}}^{2}+\mathrm{d} t^{2}\right)\right) & \rightarrow\left(\mathbb{R}^{3}-\{(0,0,0)\}, \mathrm{d} s_{\text {eucl }}^{2}\right) \\
(p, t) & \mapsto e^{t} p
\end{aligned}
$$

where $\mathbb{S}^{2}$ is the unit sphere of $\mathbb{R}^{3}$ centered at the origin. A straightforward computation shows that this map is a global isometry.

For the sake of completeness we state the following.

Corollary 12 Any twice differentiable totally umbilic hypersurface $\Sigma^{n}$ of $\mathbb{R}^{n+1}$, $n \geq 2$, is part of a hyperplane or a round sphere of $\mathbb{R}^{n+1}$.

Proof. Observe that it suffices to show that the function $\lambda$ defined as in (1) is constant. We will prove it by induction on $n \geq 2$.

For $n=2$ this is Theorem 9.

Assume the claim is true for some integer $n \geq 2$. Let $\Sigma^{n+1} \subset \mathbb{R}^{n+2}$ be a twice differentiable totally umbilic hypersurface of $\mathbb{R}^{n+2}$. Let $p \in \Sigma^{n+1}$ be any point and let $\Pi \subset \mathbb{R}^{n+2}$ be any hyperplane containing $p$ and transversal to $\Sigma^{n+1}$ at $p$. There exists $\Sigma^{n} \subset \Sigma^{n+1} \cap \Pi$ a connected subset containing $p$ which is a twice differentiable hypersurface of $\Pi$.

Let us denote by $N$ a unit normal field to $\Sigma^{n+1}$ and by $\nu$ a unit normal field along $\Sigma^{n}$ in $\Pi$. Let $v_{0} \in \mathbb{R}^{n+2}$ be a unit vector orthogonal to $\Pi$. It is easy to see that up to sign we have

$$
\left.\nu=\frac{1}{\left(1-\left\langle N, v_{0}\right\rangle^{2}\right)^{1 / 2}}\left(N-\left\langle N, v_{0}\right\rangle v_{0}\right\rangle\right)
$$

Note that $\left\langle N, v_{0}\right\rangle$ is constant along $\Sigma^{n}$. Indeed for any $u \in T \Sigma^{n}$ we have

$$
\begin{aligned}
u .\left\langle N, v_{0}\right\rangle & =\left\langle\bar{\nabla}_{u} N, v_{0}\right\rangle+\left\langle N, \bar{\nabla}_{u} v_{0}\right\rangle \\
& =\left\langle\lambda u, v_{0}\right\rangle \\
& =0 .
\end{aligned}
$$

Therefore 


$$
\begin{aligned}
\bar{\nabla}_{u} \nu & =\frac{1}{\left(1-\left\langle N, v_{0}\right\rangle^{2}\right)^{1 / 2}} \bar{\nabla}_{u} N \\
& =\frac{1}{\left(1-\left\langle N, v_{0}\right\rangle^{2}\right)^{1 / 2}} \lambda u .
\end{aligned}
$$

This shows that $\Sigma^{n}$ is a totally umbilic hypersurface in $\Pi$. By our assumption we know that $\frac{1}{\left(1-\left\langle N, v_{0}\right\rangle^{2}\right)^{1 / 2}} \lambda$ is constant and so $\lambda$ is constant along $\Sigma^{n}$.

Now varying the hyperplane $\Pi \ni p$ in $\mathbb{R}^{n+2}$ it follows that $\lambda$ is constant in a neighborhood of $p$ and so $\lambda$ is constant on the whole of $\Sigma^{n+1}$.

\section{References}

[1] Abresch, U., Rosenberg, H., A Hopf differential for constant mean curvature surfaces in $\mathbb{S}^{2} \times \mathbb{R}$ and $\mathbb{H}^{2} \times \mathbb{R}$, Acta Math. 193, No 2, (2004), 141-174.

[2] Bonahon, F., Geometric structures on 3-manifolds, Handbook of geometric topology, 93-164, North-Holland, Amsterdam, (2002).

[3] Aledo, J., Espinar, J., Gálvez, J., Complete surfaces of constant curvature in $\mathbb{H}^{2} \times \mathbb{R}$ and $\mathbb{S}^{2} \times \mathbb{R}$, to appear in Calc. Variations \& PDEs.

[4] Caddeo, R., Piu, P., Ratto, A., SO(2)-invariant minimal and constant mean curvature surfaces in 3-dimensional homogeneous spaces, Manuscripta Math, 87, (1995), 1-12.

[5] Daniel, B., Isometric immersions into $\mathbb{S}^{n} \times \mathbb{R}$ and $\mathbb{H}^{n} \times \mathbb{R}$ and applications to minimal surfaces, Preprint, (2004).

[6] Daniel, B., Isometric immersions into 3-dimensional homogeneous manifolds, to appear in Comment. Math. Helv.

[7] Do Carmo, M., Differential Geometry of Curves and Surfaces, PrenticeHall, Englewood Cliffs, NJ, (1976). 
[8] Sa Earp, R., Parabolic and hyperbolic screw motion surfaces in $\mathbb{H}^{2} \times \mathbb{R}$, Preprint, (2005).

[9] Sa Earp, R., Toubiana, R., Screw motion surfaces in $\mathbb{H}^{2} \times \mathbb{R}$ and $\mathbb{S}^{2} \times \mathbb{R}$, Illinois Journal of Math, 49, Number 4, 1323-1362, Wintner (2005).

[10] Hartman, P., Wintner, A., Umbilical points and W-surfaces, Amer. J. Math. 76, (1954), 502-508.

[11] Hopf, H., Differential Geometry in the Large, Lecture Notes in Mathematics 1000, Edited by A. Dold and B. Eckmann, (1983).

[12] Fernandez, I., Mira, P., Harmonic maps and constant mean curvature surfaces in $\mathbb{H}^{2} \times \mathbb{R}$, to appear in Amer. J. of Mathematics.

[13] Figueroa, C., Mercuri, F., Pedrosa, R., Invariant surfaces of the Heisenberg groups, Ann Mat. Pura Appl. (IV), 177, (1999), 173-194.

[14] Hauswirth, L., Generalized Riemann examples in three-dimensional manifolds, Pacific Journal of Maths, 224, No 1, (2006), 91-117.

[15] Hauswirth, L., Sa Earp, R., Toubiana, E., Associate and conjugate minimal immersions in $\mathbb{M} \times \mathbb{R}$, Preprint, (2005).

[16] Kühnel, W., Differential geometry: curves-surfaces-manifolds, 2nd edition, American Mathematical Society, Providence, RI, (2006).

[17] Meeks III, W., Rosenberg, H., The theory of minimal surfaces in $M \times R$, Comment. Math. Helv. (80), (2005), 811-858.

[18] Nelli, B., Rosenberg, H., Minimal surfaces in $H^{2} \times R$, Bull. Braz. Math. Soc. 33, (2002), 263-292.

[19] Rosenberg, H., Minimal surfaces in $\mathbb{M}^{2} \times \mathbb{R}$, Illinois J. Math. 46, $\mathrm{N}^{o} 4$, (2002), 1177-1195. 
[20] Sanini, A., Gauss map of a surface of the Heisenberg group, Boll. Un. Mat. Ital. B (7), 11, (1997), 79-93.

[21] Scott, P., The geometries of 3-manifolds, Bull. London Math. Soc., 15 (5) (1983), 401-487.

[22] Souam, R., Toubiana, E., Totally umbilic surfaces in homogeneous 3manifolds, preprint 2006, Arxiv:math.DG/0604391

[23] Spivak, M., A comprehensive introduction to differential geometry, Vol 3 and 4, Boston, Publish or Perish, (1970).

[24] Thurston, W., Three-Dimensional Geometry and Topology, Princeton, (1997).

Institut de Mathématiques de Jussieu

CNRS UMR 7586 - Université Paris VII

Case 7012, 2 Place Jussieu

75251 Paris Cedex 05, France

E-mail: souam@math.jussieu.fr

E-mail: toubiana@math.jussieu.fr 